\title{
Comparison of the NBM 200 non-invasive haemoglobin sensor with Sahli's haemometer among adolescent girls in rural India
}

\author{
A S Ahankari, ${ }^{1,2}$ J V Dixit, ${ }^{3}$ A W Fogarty, ${ }^{1}$ L J Tata, ${ }^{1}$ P R Myles ${ }^{1}$
}

\begin{abstract}
${ }^{1}$ Division of Epidemiology and Public Health, Faculty of Medicine and Health Sciences, University of Nottingham, Nottingham, UK

${ }^{2}$ Halo Medical Foundation, Andur, Maharashtra, India ${ }^{3}$ Government Medical College of Latur, Latur, Maharashtra, India
\end{abstract}

\section{Correspondence to}

Dr AS Ahankari, Halo Medical Foundation, At Post Andur, Block Tuljapur, Dist Osmanabad, Maharashtra 413603, India; dr.anandahankari@gmail.com

Received 4 May 2016 Revised 27 July 2016 Accepted 28 July 2016 Published Online First 10 August 2016

CrossMark

To cite: Ahankari AS, Dixit JV, Fogarty AW, et al. BMJ Innov 2016;2:144-148.

\begin{abstract}
Objective The study objective was to compare haemoglobin $(\mathrm{Hb})$ measurements between the NBM 200 (non-invasive Hb sensor) and Sahli's haemometer in adolescent girls in a rural Indian setting.

Methods Participants included girls aged between 13 and 17 years from 34 villages in Tuljapur and Lohara blocks of Osmanabad district, Maharashtra, India. Hb measurements from the non-invasive sensor (NBM 200) were compared with measurements obtained from Sahli's haemometer using the Bland-Altman plot, Spearman correlation coefficient, sensitivity, specificity and area under the receiver operating characteristic curve analysis (AUROC).
\end{abstract}

Results Paired measurements from both methods were obtained from 766 adolescent girls $(\mathrm{N}=766)$. Hb levels estimated by Sahli's haemometer ranged from 5.0 to $14.0 \mathrm{~g} / \mathrm{dL}$ (mean $10.1 \mathrm{~g} / \mathrm{dL}, \mathrm{SD} 1.41$ ), whereas measurements obtained from the NBM 200 ranged from 9.5 to $15.2 \mathrm{~g} / \mathrm{dL}$ (mean $12.8 \mathrm{~g} / \mathrm{dL}$, SD 1.42). The Bland-Altman analysis indicated a mean difference of $-2.70 \mathrm{~g} / \mathrm{dL}(95 \% \mathrm{Cl}-2.84$ to -2.55$)$ demonstrating an overestimation of $\mathrm{Hb}$ measurement by the NBM 200 compared with the Sahli's haemometer measurements. The NBM 200 showed low sensitivity (23.6\%) and moderate specificity (61.8\%) for the diagnosis of anaemia. The AUROC score was 0.43 indicating an underestimation of anaemia in our study population by the NBM 200.

Conclusions $\mathrm{Hb}$ measurements obtained from the NBM 200 were consistently higher leading to an underestimation of prevalence of anaemia compared with Sahli's haemometer estimates among adolescent girls in India.

\section{INTRODUCTION}

Iron deficiency anaemia (IDA) is a common nutritional disorder observed in Indian adolescent girls. ${ }^{1}$ The preliminary diagnosis of anaemia is based on haemoglobin $(\mathrm{Hb})$ levels. $^{2}$ In India, IDA prevalence among adolescent girls is about $56 \%,{ }^{2}$ which is likely to affect physical growth, psychological development, school performance and later reproductive health outcomes, particularly during pregnancy. ${ }^{3}$ Thus, early diagnosis of IDA and appropriate interventions are important in adolescent girls, especially in a country like India where marriage at a young age is followed by early pregnancies $(18.2 \%$ girls get married by the age of 15 years, and $47.4 \%$ get married by the age of 18$).{ }^{4}$ Sahli's haemometer is a widely used method for $\mathrm{Hb}$ estimation and anaemia diagnosis in rural areas of the country. ${ }^{5}$ This is an inexpensive estimation method that requires a skilled technician. However, availability of such diagnostic service is limited, particularly in geographically remote areas, where interruptions in diagnostic supplies can delay anaemia diagnosis and management. ${ }^{56}$

The NBM 200 (Orsense, Nes-Ziona, Israel) is a portable non-invasive device (operating on electricity or batteries) that provides $\mathrm{Hb}$ measurements in 60-100 s. ${ }^{7}$ Moreover, it has the advantage of not requiring material supplies (such as chemicals or needles) or post-testing biowaste management services. The device provides the benefit of non-invasive testing that is not reliant on a skilled technician in areas where diagnostic 
facilities are limited. ${ }^{8}$ The existing evidence base involves comparisons of the non-invasive technology with haematology analysers or automated finger prick devices such as HemoCue (ANGELHOLM, Sweden); however, these methods are not commonly used in rural areas in India mainly because of high operational costs and limited healthcare infrastructure. ${ }^{4} 9$ HemoCue requires a finger prick sample and is costly compared with Sahli's method due to the initial investment for the device (which ranges between GBP300 and GBP600) with higher recurring costs for testing materials compared with Sahli's approach (A Shashikant, R Kranti. Halo Medical Foundation. Personal communication. November 2015). ${ }^{10}$ Operational costs for each HemoCue test is about 3540 Indian Rupees (GBP0.35), which is twice the cost of the Sahli's method (Shashikant and Kranti. Personal communication, 2015). ${ }^{10}$ Similarly, automated haematology analysers are expensive (generally GBP4000 onwards), and require venous blood withdrawal as well as advanced laboratory infrastructure and trained personnel. A single automated analyser test costs between 100 and 350 Indian Rupees (GBP1.0-3.5; Shashikant and Kranti. Personal communication, 2015). ${ }^{10}$ While the initial cost of a non-invasive device such as NBM 200 is also high (about GBP1100), when used on a larger scale (5000 tests and above), each test costs about 15-20 Indian Rupees, which is similar with the cost of Sahli's haemometer. Moreover, the NBM 200 could be used in the community setting for mass screening by a healthcare auxiliary (Shashikant and Kranti. Personal communication, 2015). ${ }^{7}$ Therefore, we conducted a study to compare $\mathrm{Hb}$ measurements generated by the NBM 200 with Sahli's haemometer to investigate the application of the non-invasive technique in an adolescent girl population from rural areas of Maharashtra, a state of India.

\section{METHODS}

A cross-sectional survey was conducted in 34 villages of Osmanabad district of Maharashtra ( 60 000 population) to investigate the prevalence and risk factors of anaemia in adolescent girls (Maharashtra Anaemia Study, Duration 24 April 2014 to 30 June 2015). This manuscript reports on the secondary objective, which was to compare $\mathrm{Hb}$ measurements obtained from the non-invasive $\mathrm{Hb}$ sensor NBM 200 and Sahli's haemometer (the reference method in our study). The study participants comprised of adolescent girls between 13 and 17 years of age who were residents of two blocks (Tuljapur and Lohara) of Osmanabad district, and were involved in the data collection in two phases. In both phases, the study area had dry season with minimal temperature fluctuations. During the phase 1, data were collected from April to May 2014 (2 months), and in phase 2, data were collected between January and April 2015 (4 months).
Participants were recruited after obtaining written consent from the individual and their local guardian in the presence of the primary investigator (ASA). Data collection was performed at the participant's house or at the village health centre. Trained staff members collected information and performed $\mathrm{Hb}$ tests by using the two methods independently under ASA's oversight. The NBM 200 was used according to the manufacturer's guidelines, using the thumb of the non-dominant hand. 7 Sahli's haemometer was used to conduct the invasive $\mathrm{Hb}$ estimation under an aseptic protocol using the ring finger of the non-dominant hand. ${ }^{11}$ Both tests were performed in sitting position and participants were provided with written reports followed by necessary referrals to the nearest healthcare facility wherever required (based on Sahli's estimates). Anaemia was defined as a $\mathrm{Hb}$ level of $<12.0 \mathrm{~g} / \mathrm{dL} \quad(11.9 \mathrm{~g} / \mathrm{dL}$ and below), and severe anaemia was defined as a $\mathrm{Hb}$ of $7.9 \mathrm{~g} / \mathrm{dL}$ and below. ${ }^{12}$

$\mathrm{Hb}$ measurements obtained by both methods were compared using Bland-Altman analysis. ${ }^{13}$ A further comparison was performed using Spearman correlation coefficient, sensitivity, specificity, negative predictive value (NPV), positive predictive value (PPV) and area under receiver operating characteristic curve (AUROC). To allow for possible confounding by environmental temperature, a sensitivity analysis of two consecutive months was performed using the Bland-Altman analysis. All data analyses were performed using Stata Statistical Software (V.13.1). The study was approved by the Institutional Ethics Committee of Government Medical College Aurangabad, Maharashtra, India (reference number Pharma/IEC/GMA/196/2014), and was also sanctioned by the Nottingham University Medical School Ethics Committee, UK (reference number E10102013).

\section{RESULTS}

In total, 791 eligible adolescent girls from 34 villages were contacted, and we obtained paired $\mathrm{Hb}$ measurements using both methods from 766 adolescent girls in total (response rate 95.8\%). During the phase 1, data were collected from 203 participants and the rest were collected in the second phase $(\mathrm{N}=563)$. Hb estimated by Sahli's haemometer ranged from 5.0 to $14.0 \mathrm{~g} / \mathrm{dL}$ with a mean of $10.1 \mathrm{~g} / \mathrm{dL}$ (SD 1.41). The non-invasive sensor yielded $\mathrm{Hb}$ estimates in the range of $9.5-15.2 \mathrm{~g} / \mathrm{dL}$ (SD 1.42) with a mean $\mathrm{Hb}$ of $12.8 \mathrm{~g} / \mathrm{dL}$. The reference method (Sahli's haemometer) showed a high prevalence of anaemia (84\%) while, the NBM 200 diagnosed only $26 \%$ of participants (out of 766) as anaemic. The haemometer identified 41 participants $(5 \%)$ with severe anaemia $(\mathrm{Hb}$ $7.9 \mathrm{~g} / \mathrm{dL}$ and below), and the NBM 200 sensor did not identify any severe anaemic participants.

The Bland-Altman analysis showed a mean difference of $-2.70 \mathrm{~g} / \mathrm{dL}(95 \% \mathrm{CI}-2.84$ to -2.55$)$ in $\mathrm{Hb}$ 


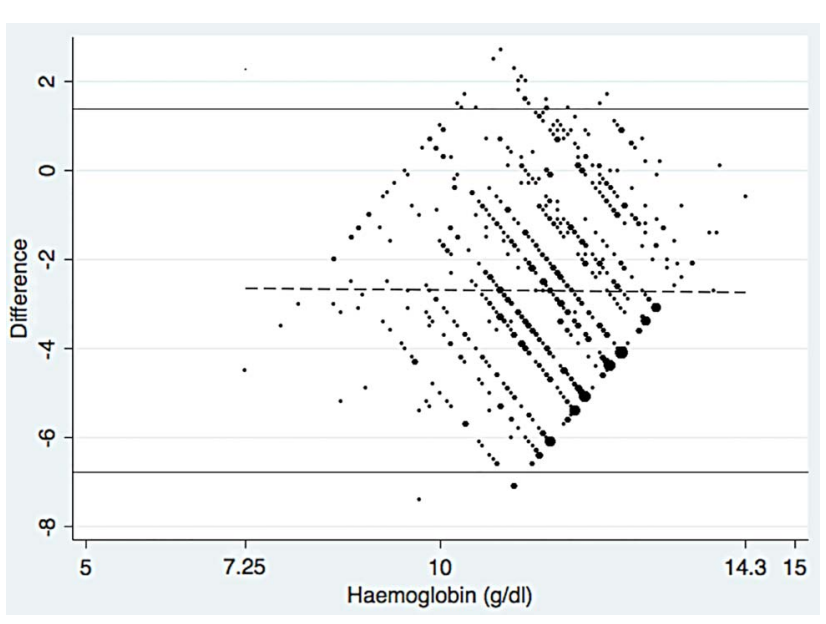

Figure 1 Bland-Altman plot. Limits of agreement -6.78 to 1.37, mean difference $-2.70(95 \% \mathrm{Cl}-2.84$ to -2.55$)$, range 7.25 to 14.30 .

estimates suggesting a systematic overestimation of $\mathrm{Hb}$ using the non-invasive technique (figure 1). The Bland-Altman plot showed a diamond-shaped pattern suggesting that there are $\sim 15$ subgroups with a linear association, between Sahli's haemometer $\mathrm{Hb}$ measurements and the difference between the two devices. Examination of this in more detail showed that this is a consequence of the difference between the two measurement techniques being similar for number of readings. A histogram plotting $\mathrm{Hb}$ estimates obtained using Sahli's method (reference method) showed a normal distribution, while the histogram of $\mathrm{Hb}$ estimates obtained using the NBM 200 was not normally distributed, and showed a plateau-like presentation (figure 2). A statistically significant weak negative correlation was found between the two methods (Spearman's test $\mathrm{r}=-0.07, \mathrm{p}=0.03$ ). The NBM 200 had low sensitivity $(23.6 \%, 95 \%$ CI $20.4 \%$ to $27.1 \%)$, moderate specificity $(61.8 \%, 95 \%$ CI $52.6 \%$ to $70.4 \%)$, low NPV $(13.4 \%, 95 \%$ CI $10.7 \%$ to $16.5 \%)$ and moderate PPV $(76.4 \%, 95 \%$ CI $69.9 \%$ to

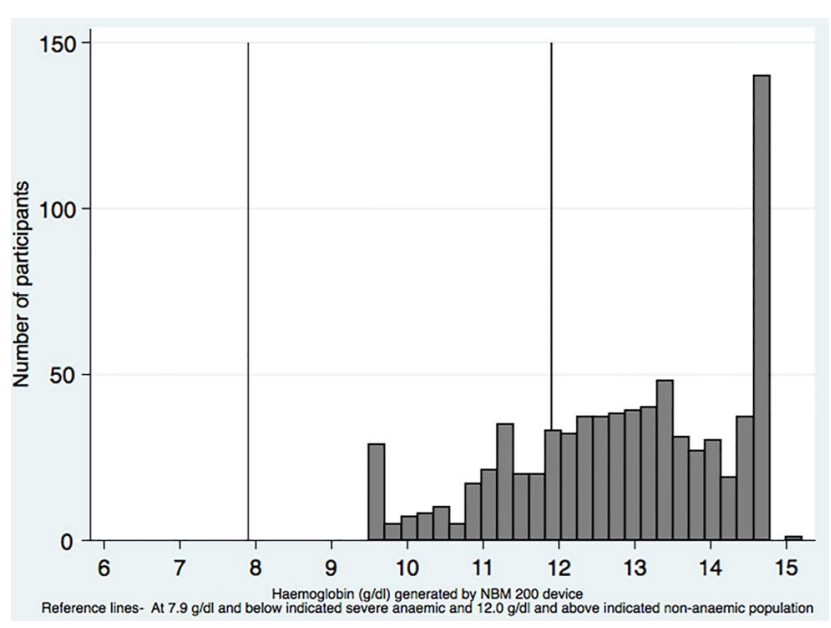

Figure 2 Histogram of NBM 200 measurements.
82.1\%). The AUROC score was 0.43 suggesting poor anaemia diagnostic performance of the non-invasive sensor in our study population. Sensitivity analysis of a subgroup of data collection over 2 months (April to May) resulted in a mean difference of $-0.51 \mathrm{~g} / \mathrm{dL}$ with wide limits of agreement ( -3.46 to $2.43 \mathrm{~g} / \mathrm{dL}$ ), demonstrating a consistent overestimation of $\mathrm{Hb}$ measurement by NBM 200 compared with the haemometer.

\section{DISCUSSION}

This study consisting of adolescent girls aged 1317 years from rural India found that the non-invasive sensor NBM 200 overestimated $\mathrm{Hb}$ when compared with Sahli's haemometer. The sensor underestimated anaemia prevalence (sensitivity $23.6 \%$ ), and failed to detect severe anaemia cases in our study population.

\section{Strengths and limitations}

To the best of our knowledge, this is the first study comparing the non-invasive technology with Sahli's haemometer ${ }^{9}{ }^{14}$ and also provided an opportunity to compare the technique in remote locations, where the Sahli's haemometer is currently used. The study had a good response rate $(95.8 \%)$, and none of the study participants had thumb injury, ulceration or any skin colourants (such as Henna) thus allowing for the noninvasive measurements to be obtained in accordance with the guidelines. ${ }^{15}$

Nevertheless, our study had certain limitations. Sahli's haemometer is not a gold standard method for measuring $\mathrm{Hb}$ and may be susceptible to human error, but we were unable to perform additional measurements using the cyanmethaemoglobin technique or an automated haematology analyser (gold standard) due to logistical challenges involving transportation and hence integrity of samples collected in a hot remote rural setting. ${ }^{10} 14$ However, we optimised the quality of the haemometer measurements to provide the best data available in this environment. First, the technician involved in the MAS study had 4 years of laboratory experience, and received training provided by the primary investigator and the Halo Medical Foundation (HMF) hospital consultant. Additionally, during data collection, two study members (technician and the primary investigator) agreed on the Sahli's estimation for each participant to minimise ascertainment bias. We did not find any major differences between the two members $(<0.2 \mathrm{~g} / \mathrm{dL})$. It is important to note that venous blood withdrawal is not routinely used for anaemia screening in the adolescent population in our study setting, and insisting on this for blood investigation using an automated analyser, may have adversely affected our response rate. Future studies in this area should consider using a gold standard reference measurement for $\mathrm{Hb}$ if possible. Second, the Bland-Altman plot formed a diamondshaped pattern suggesting that the difference between 
the two $\mathrm{Hb}$ measurement techniques was highly variable. The pattern is likely to be due to technique variability (which could affect measurements obtained from either the NBM 200 or Sahli's haemometer). The variability was more apparent for more frequently occurring $\mathrm{Hb}$ values (for, eg, 94 participants had an $\mathrm{Hb}$ value of $9.5 \mathrm{~g} / \mathrm{dL}$ ) and less apparent for rarer $\mathrm{Hb}$ values, resulting in the diamond-shaped plot.

\section{Synthesis}

While we have used Sahli's haemometer as the reference method, the results are in agreement with our another study comparing the NBM 200 with the automated haematology analyser (Sysmex XP-100, Japan) in pregnant women in the same setting $(\mathrm{N}=269) .{ }^{14}$ Results from the pregnant women study showed that the NBM 200 overestimated Hb levels with subsequent underestimation of anaemia status (sensitivity of 33.7\%). ${ }^{14}$ Findings from the pregnant women study showed a mean difference of $-1.8 \mathrm{~g} / \mathrm{dL}$ (95\% CI -2.06 to -1.71$)$ when assessed using Bland-Altman method, indicating an overestimation of $\mathrm{Hb}$ by the NBM 200, which is in agreement with our current adolescent girl analysis. To the best of our knowledge, there is no published evidence comparing the NBM 200 and Sahli's haemometer. A study evaluated the finger prick technique using HemoCue (ANGELHOLM, Sweden) with NBM 200 in blood donation centres/hospitals. ${ }^{16}$ The HemoCue uses peripheral capillary blood similar to the Sahli's haemometer, but is considered to have better precision because of reduced human error. ${ }^{17}$ However, Kim et $a l^{16}$ showed that compared with an automated haematology analyser (LH500, Beckman Coulter, Brea, California, USA), both the NBM 200 and HemoCue showed low sensitivity (38\% and $47 \%$, respectively). We acknowledge that Sahli's haemometer estimates are susceptible to interpretation; however, given that this is a commonly used technique in the country, it was important to compare the NBM 200 to Sahli's haemometer. The NBM 200 failed to detect severe anaemia in our study population similar to our findings from pregnant women participants, ${ }^{14}$ and thus cannot be used as an alternative to the current diagnostic technique. However, if improved substantially or recalibrated by the manufacturer in near future to provide precise measurements in an Indian setting, non-invasive technology may be useful for anaemia screening in remote and also in urban areas to facilitate early diagnosis and management.

\section{CONCLUSION}

This research is the first to provide a comparison between Sahli's haemometer and the NBM 200 in developing country settings. The ability to obtain $\mathrm{Hb}$ measurements involving a large representative adolescent girl population from difficult to reach communities is a major strength of the study. We report a systematic overestimation of $\mathrm{Hb}$ using the NBM 200, which is consistent with our study involving pregnant women in the same geographical location in India. There is an urgent need to improve the non-invasive technology, as it will provide opportunities for mass screening in a country having a high prevalence of anaemia. Future studies of improved non-invasive $\mathrm{Hb}$ devices will be required in developing countries to validate this technology before it is used independently for anaemia diagnosis and treatment.

Twitter Follow Anand Ahankari at@AnandAhankari

Acknowledgements The authors acknowledge the support of Ms Sandhya Rankhamb in data collection, data entry, verification and recognise her contribution in the project. They thank the Halo Medical Foundation (HMF) village health workers for providing field-level support for this study. They dedicate their research to Dr Sulbha Hardikar and Professor (Mr) and Mrs Chawathe who supported the project and the PhD studies, respectively.

Contributors The study was designed by AWF, PRM, LJT and ASA. ASA obtained the data and ASA, AWF and PRM conducted the analysis. All authors (ASA, PRM, AWF, JVD and LJT) participated in manuscript preparation and approved the final manuscript for submission.

Funding This study was conducted as part of ASA's PhD programme with the University of Nottingham UK, which was sponsored by the University's Vice Chancellor Scholarship for Research Excellence International 2013 (Tuition fee support, Reference 12031). The anaemia project conducted in Maharashtra, India, was a joint collaboration between the university and HMF, with the latter providing laboratory testing and data storage facilities. Project management and data collection were funded by Dr Hardikar through the Maharashtra Foundation, USA. Professor (Mr) and Mrs Chawathe, Mumbai, India, provided generous support for ASA's study. ASA also received a bursary from the Durgadevi Charitable Trust, India, during the $\mathrm{PhD}$ programme. The non-invasive NBM 200 unit was sponsored by the Clinical Translational Research Priority Group Award for International Collaboration, University of Nottingham, UK (grant code A2RN72).

Competing interests None declared.

Ethics approval The study was approved by the Institutional Ethics Committee of the Government Medical College Aurangabad, Maharashtra, India (reference number Pharma/ IEC/GMA/196/2014), and the Medical School Ethics Committee of the University of Nottingham, UK (reference number E10102013).

Provenance and peer review Not commissioned; externally peer reviewed.

\section{REFERENCES}

1 Singh RK, Patra S. Extent of anaemia among preschool children in EAG States, India: a challenge to policy makers. Anemia 2014;2014:868752.

2 Jawarkar A, Lokare P, Kizhatil A, et al. Prevalence of anemia and effectiveness of iron supplementation in anemic adolescent school girls at Amravati City (Maharashtra). J Health Res Rev 2015;2:7.

3 Joshi M, Gumashta R. Weekly iron folate supplementation in adolescent girls-an effective nutritional measure for the management of iron deficiency anaemia. Glob J Health Sci 2013;5:188-94. 


\section{MEDICAL DEVICES}

4 World Health Organization, South-East Asia Regional Information. http://www.searo.who.int/en/ (accessed 21 Oct 2015).

5 Kulkarni M, Durge P, Kasturwar N. Prevalence of anemia among adolescent girls in an urban slum. Natl J Community Med 2012;3:108-11.

6 Pasricha SR, Biggs BA, Prashanth NS, et al. Factors influencing receipt of iron supplementation by young children and their mothers in rural India: local and national cross-sectional studies. BMC Public Health 2011;11:617.

7 OrSense-Non-Invasive Technologies. http://www.orsense.com/ (accessed 21 Oct 2015).

8 Noninvasive technology for anemia detection. http://www.path. org/publications/files/TS_update_anemia.pdf (accessed 21 Oct 2015).

9 Kim SH, Lilot M, Murphy LS, et al. Accuracy of continuous noninvasive hemoglobin monitoring. Anesth Analg 2014;119:332-46.

10 Srivastava T, Negandhi H, Neogi S, et al. Methods for hemoglobin estimation: a review of "What Works". J Hematol Tranfusion Med 2014;2:1025-8.

11 Yoshida A, Saito K, Ishii K, et al. Assessment of noninvasive, percutaneous hemoglobin measurement in pregnant and early postpartum women. Med Devices (Auckl) 2014;7:11-16.

12 Anaemia Guidelines-Government of India. http://nrhm.gov.in/ nrhm-components/rmnch-a/child-health-immunization/ child-health/guidelines.html (accessed 21 Oct 2015).

13 Bland JM, Altman DG. Statistical methods for assessing agreement between two methods of clinical measurement. Lancet 1986;1:307-10.

14 Ahankari AS, Fogarty AW, Tata LJ, et al. Assessment of a non invasive haemoglobin sensor NBM 200 among pregnant women in rural India. BMJ Innov 2016;2:70-7.

15 Ardin S, Störmer M, Radojska S, et al. Comparison of three noninvasive methods for hemoglobin screening of blood donors. Transfusion 2015;55:379-87.

16 Kim MJ, Park Q, Kim MH, et al. Comparison of the accuracy of noninvasive hemoglobin sensor (NBM-200) and portable hemoglobinometer (HemoCue) with an automated hematology analyzer (LH500) in blood donor screening. Ann Lab Med 2013;33:261-7.

17 Shah PP, Desai SA, Modi DK, et al. Assessing diagnostic accuracy of Haemoglobin Colour Scale in real-life setting. J Health Popul Nutr 2014;32:51-7. 